07

\title{
Низкочастотный магнитоэлектрический датчик без смещения на основе слоистой структуры Metglas/кварц/Metglas
}

\author{
(C) Changxing Sun ${ }^{1}$, Wenrong Yang ${ }^{1, \uparrow, ~ Y i f a n ~} \mathrm{He}^{2}$, Lei Chen ${ }^{2}$, Cunzheng Dong ${ }^{2}$, \\ Xianfeng Liang ${ }^{2}$, Huaihao Chen ${ }^{2}$, Nian-Xiang Sun ${ }^{2}$ \\ ${ }^{1}$ State Key Laboratory of Reliability and Intelligence of Electrical Equipment, Hebei University of Technology, Tianjin, China \\ ${ }^{2}$ Department of Electrical and Computer Engineering, Northeastern University, Boston, Massachusetts, USA \\ 『E-mail: wryang@hebut.edu.cn
}

Поступило в Редакцию 7 апреля 2021 г.

В окончательной редакции 11 июня 2021 г.

Принято к публикации 13 июня 2021 г.

К настоящему времени достигнут огромный прогресс в повышении эффективности магнитоэлектрических датчиков, предназначенных для регистрации низкочастотных магнитных полей путем умножения частоты, которые строятся на основе магнитоэлектрических композитов. Предлагается новая конструкция магнитоэлектрического датчика, построенная на основе слоистой композитной структуры аморфный металл Metglas/кристалл пьезоэлектрического кварца/аморфный металл Metglas. Фольга Metglas приклеивается эпоксидной смолой симметрично по обе стороны пластины кристаллического кварца, вырезанной перпендикулярно оси $X$. Эксперименты показали, что при использовании метода умножения частоты можно добиться предела чувствительности композитного магнитоэлектрического датчика к низкочастотному магнитному полю в $11 \mathrm{pT}$ при частоте $1 \mathrm{~Hz}$ при полном отсутствии поля смещения. Испытания также показали, что при частоте $1 \mathrm{~Hz}$ спектральная плотность мощности шума датчика составляет $3.93 \cdot 10^{-6} \mathrm{~V} / \mathrm{Hz}^{1 / 2}$. Из полученных результатов видно, что предлагаемый датчик обладает рядом благоприятных характеристик, обеспечивающих экономичный и высокоэффективный метод измерения низкочастотного магнитного поля.

Ключевые слова: низкочастотный, предел чувствительности, магнитоэлектрический датчик, кристалл пьезоэлектрического кварца.

DOI: 10.21883/PJTF.2021.19.51503.18814

В последние годы проводились интенсивные исследования магнитоэлектрических (МЭ) материалов, которые вызывают интерес благодаря широкому спектру областей их применения: магнитоэлектрические датчики, механические антенны, запоминающие и настраиваемые микроволновые устройства и пр. [1-4]. МЭ-композиты, изготовленные из пьезоэлектрических и магнитострикционных материалов, могут обеспечивать более высокое МЭ-напряжение, чем сложные однофазные мультиферроидные материалы. Для достижения высокой эффективности МЭ-гетероструктур было предложено также использовать различные виды композитных материалов, такие как композиты с порошковым наполнением или слоистые композиты, которые применяются при различных режимах вибрации. Область применения магнитоэлектрических композитов с порошковым наполнителем ограничена по причине их относительно слабой механической связи, низкого МЭ-коэффициента и высоких диэлектрических потерь [5]. В отличие от них слоистые МЭ-композиты обладают более высоким МЭ-коэффициентом, а также более простой структурой, благодаря чему заметно упрощается процесс их изготовления. На структуре из монокристаллического волокна $\mathrm{PMN}-\mathrm{PT}$, ламинированного с помощью эпоксидного клея аморфным сплавом $\mathrm{FeBSi}$, обработанным лазерным облучением, при низких температурах можно получить высокий магнитоэлектрический коэффициент на частоте резонанса, достигающий значения $7000 \mathrm{~V} /(\mathrm{cm} \cdot \mathrm{Oe})$ [6]. Трехслойные ламинатные МЭ-датчики обладают высоким МЭ-коэффициентом при резонансной частоте в килогерцевом диапазоне, в то время как для регистрации магнитных полей меньшей частоты предлагаются двухслойные структуры МЭ-датчиков с резонансной частотой изгибных колебаний в несколько сотен $\mathrm{Hz}$ [7].

Тем не менее регистрация биомагнитных сигналов в пикотесловом диапазоне или ниже при низких частотах от 1 до $100 \mathrm{~Hz}$ всегда является весьма сложной задачей из-за высокого уровня шума, пропорционального $1 / f$. В настоящее время для регистрации низкочастотных биомагнитных сигналов при экстремально низких рабочих температурах применяются высокочувствительные сверхпроводящие квантовые интерферометрические детекторы (СКВИД). Перспективной альтернативой СКВИД в задаче регистрации биомагнитных сигналов являются высокочувствительные и компактные МЭ-датчики [8]. Чтобы иметь возможность усилить магнитоэлектрический эффект слоистого МЭ-датчика, пьезоэлектрическая фаза должна обладать высоким пьезоэлектрическим коэффициентом и низкой диэлектрической проницаемостью. По сравнению с пьезоэлектрическими преобразователями монокристаллический пьезоэлектрический кварц обладает более выгодными свойствами, отношение его пьезоэлектрического модуля к диэлектрической проницаемости 
составляет $0.05 \mathrm{~m}^{2} / \mathrm{C}[9,10]$. Кроме того, высокая добротность устройств на основе кварца обеспечивает отсутствие в них эффектов ферроэлектрического гистерезиса и пироэлектрических потерь.

В настоящей работе предлагается инновационный магнитоэлектрический датчик с трехслойной структурой, состоящей из кварцевого кристалла, вырезанного перпендикулярно оси $X$, и фольги Metglas. Сигнал низкочастотного магнитного поля генерируется и регистрируется кварцевым датчиком с использованием схемы частотной модуляции. Предел чувствительности МЭ-датчика определялся в отсутствие смещения по постоянному току.

При изготовлении датчика пластина кварца толщиной $500 \mu \mathrm{m}$, вырезанная перпендикулярно оси $X$ и отполированная с двух сторон, разрезалась на пластины размером $72 \times 9 \mathrm{~mm}$. В качестве электродов на обе стороны пластины наносились методом магнетронного распыления тонкие $(100 \mathrm{~nm})$ пленки платины. Пленки фольги из аморфного металла Metglas толщиной $21 \mu \mathrm{m}$ с высокой магнитострикционной способностью разрезались на кусочки размером $70 \times 9 \mathrm{~mm}$. Десять слоев фольги Metglas укладывались друг на друга и симметрично приклеивались эпоксидным клеем по обе стороны кварцевой пластинки. Слои Metglas выравнивались по кромке одной длинной стороны кварцевой пластинки. И наконец, слоистая структура Metglas/кварц/Metglas укладывалась в вакуумную упаковку и выдерживалась в течение $24 \mathrm{~h}$ при комнатной температуре для того, чтобы уменьшить до минимума толщину эпоксидной пленки между слоями. Готовое устройство устанавливалось в самодельную модуляционную катушку для проведения низкочастотных измерений.

При этом из-за высокой плотности мощности шума и низкого МЭ-коэффициента в низкочастотном диапазоне для регистрации низкочастотного магнитного поля напряженностью менее 100 pТ необходимо использовать частотное преобразование. Если принята эта схема, то генерация модулированной частотной составляющей происходит вблизи резонансной области, причем интенсивность составляющей пропорциональна интенсивности регистрируемого низкочастотного магнитного сигнала. В том случае, если к МЭ-датчику прикладывается магнитное поле небольшой напряженности $H$, коэффициент прямого МЭ-взаимодействия $\alpha_{\mathrm{ME}}$ задается формулой $\alpha_{\mathrm{ME}}=\partial V_{\mathrm{ME}} / \partial H$. Величина $V_{\mathrm{ME}}$ представляет собой напряжение, индуцированное в пьезоэлектрической фазе, которой передается напряжение деформации магнитострикционных Metglas-слоев. Магнитострикционную реакцию аморфной ленты Metglas можно описать с помощью модели Ливингстона для когерентного вращения намагниченности. В рамках модели Ливингстона [11] напряжение деформации магнитострикционного материала описывается формулой $S=\mathrm{CH}^{2}$. Здесь $H$ - напряженность приложенного магнитного поля, $C$ - магнитострикционный параметр. Если суммарное магнитное поле, приложенное к датчику, можно представить как $H=H_{\text {mod }}(t)+H_{s i g}(t)$, то деформация магнитострикционного материала описывается формулой

$$
S=C\left(H_{\text {sig }}(t)^{2}+2 H_{\text {sig }}(t) H_{\text {mod }}(t)+H_{\text {mod }}(t)^{2}\right),
$$

где $H_{\text {mod }}$ - модулирующий сигнал, а $H_{s i g}-$ регистрируемый слабый сигнал переменного тока. Если выходной сигнал датчика с магнитной модуляцией обработать полосовым пропускающим фильтром или синхронизирующим усилителем, его формула принимает следующий вид:

$$
S=2 C H_{\text {sig }}(t) H_{\text {mod }}(t)
$$

Если

$H_{\text {sig }}(t)=H_{\text {sig }} \cos \left(2 \pi f_{\text {sig }} t\right)$ и $H_{\text {mod }}(t)=H_{\text {mod }} \cos \left(2 \pi f_{\text {mod }} t\right)$,

деформация описывается следующим образом:

$$
\begin{aligned}
2 H_{\text {sig }}(t) H_{\text {mod }}(t)= & 2 H_{\text {mod }} H_{\text {sig }} \cos \left(2 \pi f_{\text {mod }} t\right) \cos \left(2 \pi f_{\text {sig }} t\right) \\
= & H_{\text {mod }} H_{\text {sig }} \cos \left(2 \pi\left(f_{\text {mod }}+f_{\text {sig }}\right) t\right) \\
& +H_{\text {mod }} H_{\text {sig }} \cos \left(2 \pi\left(f_{\text {mod }}-f_{\text {sig }}\right) t\right)
\end{aligned}
$$

где $f_{\text {mod }}$ и $f_{s i g}$ - частоты модулирующего сигнала и слабого сигнала переменного тока соответственно. В качестве модулирующей частоты $f_{\text {mod }}$ выбирается резонансная частота датчика, благодаря чему обеспечивается его высокая чувствительность в низкочастотном режиме.

Датчик помещается в центр системы из пары катушек Гельмгольца. Катушки Гельмгольца создают низкочастотный магнитный сигнал. Самодельная катушка, намотанная на датчик, играет роль источника модулирующего сигнала. Для измерения выходного сигнала используется анализатор сигнала SR785 производства Stanford Research Systems. Для устранения электромагнитных помех, которые могли бы затруднить регистрацию низкочастотных магнитных полей, испытательная установка помещалась в цилиндрическую камеру, экранирующую магнитное поле.

Композитные МЭ-датчики требуют наличия внешнего магнитного поля смещения для усиления магнитоэлектрического эффекта, что увеличивает их размеры и стоимость. В настоящей работе испытания кварцевого датчика с намагниченным аморфным металлом Metglas проводились в отсутствие магнитного смещения постоянного тока. Резонансная частота датчика измерялась импедансным анализатором Agilent 4194А и оказалась равной $34.72 \mathrm{kHz}$. Поэтому для того чтобы получить возможность регистрировать низкочастотное $(1 \mathrm{~Hz})$ магнитное поле, к композитным МЭ-датчикам прикладывалось несущее поле частотой $34.72 \mathrm{kHz}$. Спектры выходного напряжения МЭ-датчика представлены на рис. 1. 
$a$

$b$
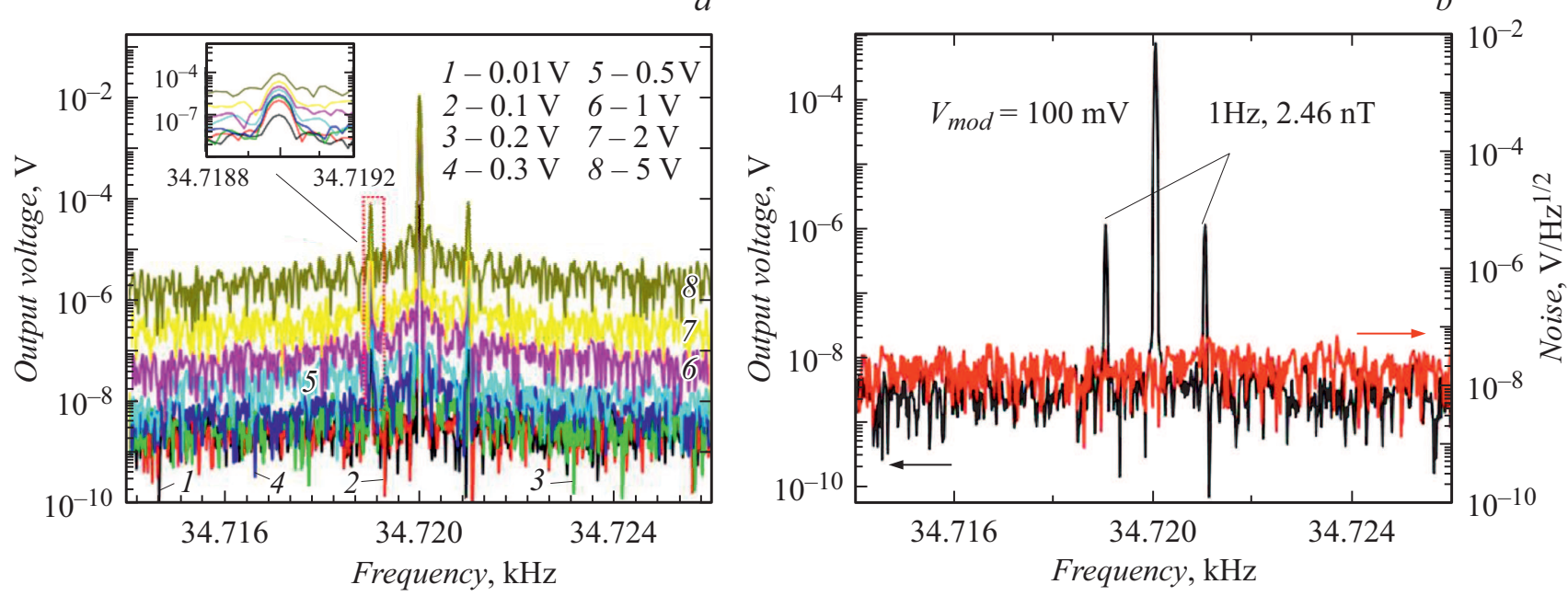

Рис. 1. Данные измерений модулирующего спектра и шумового сигнала. $a$ - зависимость наведенного напряжения МЭ-датчика при различных модулирующих напряжениях. $b-$ спектр выходного напряжения и шумового сигнала.

На рис. 1, $a$ показаны спектры выходного напряжения МЭ-датчика при различных модулирующих полях переменного тока. Спектр содержит три пика: один соответствующий частоте модуляции $f_{\text {mod }}$, и два пика одинаковой амплитуды, расположенные по бокам от него, $-f_{\text {mod }} \pm f_{\text {sig. }}$. На вставке к рис. 1, $a$ боковой пик, расположенный на стороне более низких частот, показан детальнее. Наведенное напряжение при частоте $34.721 \mathrm{kHz}$ растет с увеличением приложенного модулирующего напряжения. К сожалению, интенсивность шума подчиняется тому же закону, что и наведенное (индуцированное) напряжение. С целью оптимизации соотношения сигнал/шум было выбрано $V_{\text {mod }}=100 \mathrm{mV}$, соответствующее напряженности модулирующего магнитного поля $H_{m o d}=2$ Ое. На рис. $1, b$ представлен спектр выходного напряжения МЭ-датчика, регистрирующего магнитное поле при частоте $1 \mathrm{~Hz}$ и индукции $2.46 \mathrm{nT}$, с оптимизированной амплитудой модулирующего напряжения и спектральной плотностью мощности шума в области резонансной частоты. Пиковое наведенное напряжение низкочастотной боковой полосы составляет $1.06 \mu \mathrm{V}$, в то время как уровень шумового напряжения равняется $2.61 \mathrm{nV}$. При магнитном поле переменного тока, характеризующемся величиной $2.46 \mathrm{nT}$, наблюдаемое отношение сигнал/шум составляло $52.3 \mathrm{~dB}$. Таким образом, расчет предела чувствительности дает величину порядка 6 рТ. На рис. 2 представлена зависимость от магнитного поля реального низкочастотного предела чувствительности датчика при использовании метода частотной модуляции.

При уменьшении величины $H_{s i g}$ сначала наблюдается линейное снижение выходного напряжения МЭ-датчика от нанотесловых до пикотесловых значений индукции низкочастотного магнитного поля переменного тока. Затем выходное напряжение датчика перестает коррелировать с величиной $H_{s i g}$, в основном потому, что
МЭ-сигнал переменного тока сильно зависит от уровня шумового напряжения. Точка пересечения кривых, в которой соотношение сигнал/шум равно 1, и является пределом чувствительности датчика. Значения предела чувствительности были измерены также при частотах, равных 10 и $100 \mathrm{~Hz}$. Из рис. 2 видно, что пределы чувствительности составляют 11,16 и 22 рТ при 1 , 10 и $100 \mathrm{~Hz}$ соответственно. Такие значения предела чувствительности характерны для данного типа классических симметричных структур резонансных датчиков. Чувствительность кварцевого датчика при $1 \mathrm{~Hz}$ выше, чем при 10 и $100 \mathrm{~Hz}$. Это может быть связано с высокой добротностью кварца, благодаря которой при использовании метода частотной модуляции усиление резонанса ограничивается очень узким диапазоном частот, максимум в несколько Нz. Это используется для регистрации

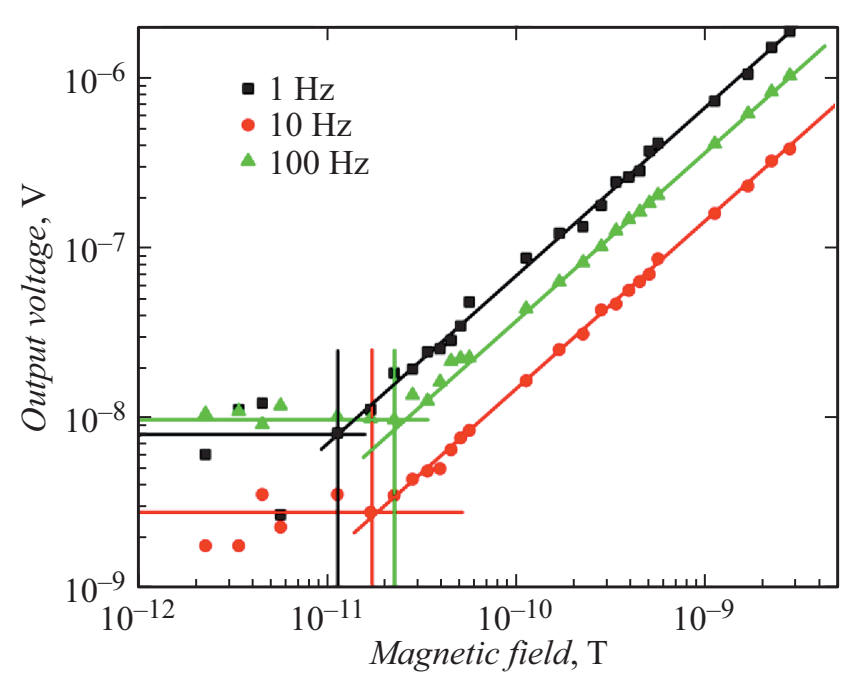

Рис. 2. Предел чувствительности датчика при различных значениях частоты. 


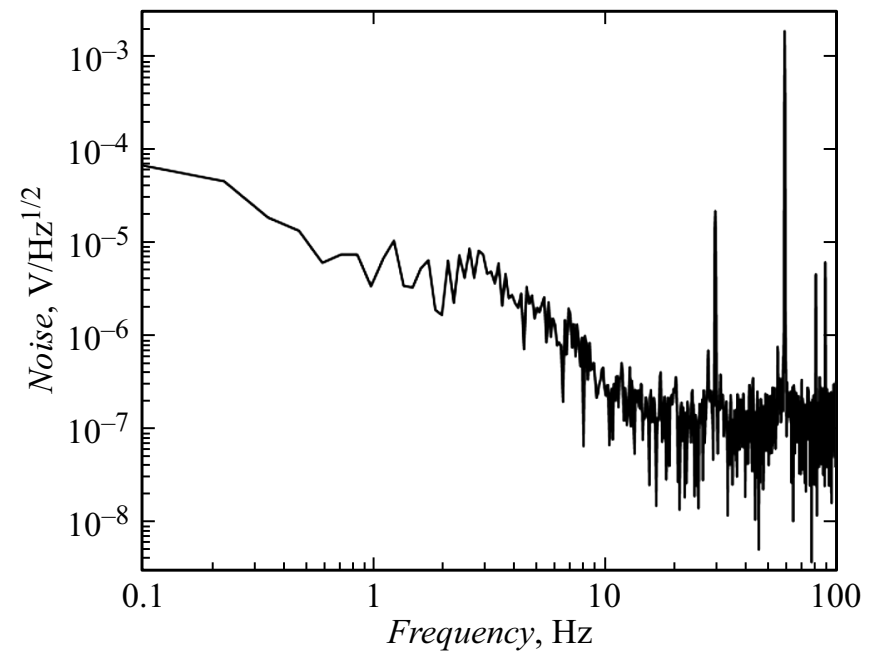

Pис. 3. Данные измерений спектральной мощности шума МЭ-датчика.

пикотесловых полей при низких частотах $(1-100 \mathrm{~Hz})$ и комнатной температуре. Шумы датчика измерялись при комнатной температуре с помощью анализатора частотных спектров, внешнее магнитное поле при этом к датчику не прикладывалось.

На рис. 3 представлены результаты измерения спектральной мощности шума датчика в диапазоне от 0.1 до $100 \mathrm{~Hz}$. Для устранения внешних помех измерения проводились в экранирующей камере. Из рисунка видно, что основная составляющая шума приходится на частоту $60 \mathrm{~Hz}$, а также на частоты, кратные этой величине. Оказалось, что плотность шума при $1 \mathrm{~Hz}$ составляет $3.93 \cdot 10^{-6} \mathrm{~V} / \mathrm{Hz}^{1 / 2}$. Низкочастотный магнитный сигнал амплитудой 11 рТ при $1 \mathrm{~Hz}$ может быть зарегистрирован с использованием частотного преобразования без смещения. Уровень шума спектрального анализатора SR780 значительно ниже измеренного уровня шума, причем эта разница достаточна для того, чтобы влияние шума на результаты измерений отсутствовало.

Таким образом, возможность изготовления МЭ-датчика с трехслойной структурой можно считать доказанной. В работе исследовалась возможность эксплуатации предложенного МЭ-датчика для регистрации низкочастотного магнитного поля с использованием метода частотного преобразования. Экспериментальные результаты показали, что при использовании метода частотного преобразования предел чувствительности датчика к слабому магнитному полю низок и составляет 11, 16 и $22 \mathrm{pT}$ при частотах 1,10 и $100 \mathrm{~Hz}$ соответственно. Кроме того, возможность работы датчика в отсутствие магнитного поля смещения позволяет уменьшить его размеры. Поэтому такие датчики весьма перспективны в области измерения промышленных магнитных полей в тех случаях, когда размеры и вес измерительных приборов могут играть существенную роль.

\section{Финансирование работы}

Финансовая поддержка исследования осуществлялась State Key Laboratory of Reliability and Intelligence of Electrical Equipment (N EERI_PI2020004), National Natural Science Foundation of China (51877066) и Joint Doctoral Training Foundation of HEBUT (2018HW0002).

\section{Конфликт интересов}

Авторы заявляют, что у них нет конфликта интересов.

\section{Список литературы}

[1] J. Zhai, Z. Xing, S. Dong, J. Li, D. Viehland, J. Am. Ceram. Soc., 91 (2), 351 (2008). DOI: $10.1111 / \mathrm{j} .1551-2916.2008 .02259 . \mathrm{x}$

[2] C. Dong, Y. He, M. Li, C. Tu, Z. Chu, X. Liang, H. Chen, Y. Wei, M. Zaeimbashi, X. Wang, H. Lin, Y. Gao, N.X. Sun, IEEE Antennas Wirel. Propag. Lett., 19 (3), 398 (2020). DOI: 10.1109/LAWP.2020.2968604

[3] В.Л. Преображенский, Л.М. Крутянский, N. Tiercelin, P. Pernod, Письма в ЖТФ, 46 (1), 43 (2020). DOI: $10.21883 /$ PJTF.2020.01.48864.18056 [V.L. Preobrazhensky, L.M. Krutyansky, N. Tiercelin, P. Pernod, Tech. Phys. Lett., 46 (1), 38 (2020). DOI: 10.1134/S1063785020010113].

[4] X. Liang, C. Dong, H. Chen, J. Wang, Y. Wei, M. Zaeimbashi, Y. He, A. Matyushov, C. Sun, N.X. Sun, Sensors, 20 (5), 1532 (2020). DOI: $10.3390 / \mathrm{s} 20051532$

[5] Z. Chu, M. PourhosseiniAsl, S. Dong, J. Phys. D: Appl. Phys., 51 (24), 243001 (2018). DOI: 0.1088/1361-6463/aac29b

[6] Z. Chu, H. Shi, W. Shi, G. Liu, J. Wu, J. Yang, S. Dong, Adv. Mater., 29 (19), 1606022 (2017).

DOI: 10.1002 /adma.201606022

[7] J. Ou-Yang, X. Liu, H. Zhou, Z. Zou, Y. Yang, J. Li, Y. Zhang, B. Zhu, S. Chen, X. Yang, J. Phys. D: Appl. Phys., 51 (32), 324005 (2018). DOI: 10.1088/1361-6463/aaced8

[8] S. Zuo, J. Schmalz, M. Ozden, M. Gerken, J. Su, F. Niekiel, F. Lofink, K. Nazarpour, H. Heidari, IEEE Trans. Biomed. Circuits Syst., 14 (5), 971 (2020). DOI: 10.1109 /TBCAS.2020.2998290

[9] В.М. Лалетин, Д.А. Филиппов, Н.Н. Поддубная, И.Н. Маничева, G. Srinivasan, Письма в ЖТФ, 45 (9), 16 (2019). DOI: 10.21883/PJTF.2019.09.47706.17661 [V.M. Laletin, D.A. Filippov, N.N. Poddubnaya, I.N. Manicheva, G. Srinivasan, Tech. Phys. Lett., 45 (5), 436 (2019). DOI: $10.1134 / \mathrm{S} 1063785019050092]$.

[10] В.М. Лалетин, Д.А. Филиппов, С.Е. Мозжаров, И.Н. Маничева, Письма в ЖТФ, 44 (7), 16 (2018). DOI: 10.21883 /PJTF.2018.07.45880.17103 [V.M. Laletin, D.A. Filippov, S.E. Mozzharov, I.N. Manicheva, Tech. Phys. Lett., 44 (4), 281 (2018). DOI: $10.1134 / \mathrm{S} 1063785018040065]$.

[11] J.D. Livingst, Phys. Status Solidi A, 70 (2), 591 (1982). DOI: $10.1002 /$ pssa.2210700228 